\title{
ON COMPLEX EXPONENTIAL GROUPS
}

\author{
Martin Moskowitz and Richard Sacksteder ${ }^{1}$
}

\begin{abstract}
Lai, Wüstner and Chatterjee have shown in a variety of cases that the exponential map of a connected complex Lie group is surjective only if its center is connected. Here we prove this is true without restriction for any complex connected Lie group.
\end{abstract}

\section{Introduction}

A connected Lie group, $G$ is said to be exponential if its exponential map, $\exp _{G}$ : $\mathfrak{g} \rightarrow G$, is surjective, or equivalently if each point of $G$ lies on a 1-parameter subgroup. Here the corresponding lower case Gothic letter denotes the Lie algebra of a Lie group. $E(G)$ will denote the range of $\exp _{G}$, so $G$ is exponential if $G=E(G)$. The question of which connected Lie groups are exponential has been studied for many years. A very useful survey of results on exponentiality until 1997 is to be found in the article by Djokovic and Hofmann, [5]. For the more recent literature and to see some of the additional complexities in the case of real groups the reader is referred to [14] and [15].

Our concern here is with complex Lie groups. It is well known that the groups $\operatorname{PSL}(n, \mathbb{C})$ are exponential and have trivial centers. Wüstner [20] and Lai [9], [10] have shown that these are the only complex simple exponential groups and therefore all complex semisimple exponential groups have trivial centers. Using maps raising elements to integer powers Chatterjee [3] proves for an algebraic group $G$ defined over an algebraically closed field of characteristic zero that if $G$ is exponential its center is connected. Our main result, Theorem 1 below, extends this fact to arbitrary complex Lie groups (and can be regarded as completing a program of Wüstner's [20]). The corresponding statement is false in the case of real groups. Thus being exponential is in some ways a more restrictive condition in the complex case than in the real case. In [12] sufficient conditions for exponentiality are determined when $G$ is a complex algebraic group (these work as they stand for any complex connected Lie group) and in fact our present result is a generalization of Theorem 11 in [12].

Our purpose here is to prove:

Theorem 1. A connected complex Lie group is exponential if and only if its center, $Z(G)$, is connected and its adjoint group, $\operatorname{Ad}(G)$, is exponential.

In the course of the proof of Theorem 1 we also get Corollary 1.1 below (see Section 2 for the definition of semi-algebraic). This follows from Proposition 5.1

Received by the editors November 8, 2007.

${ }^{1}$ Richard Sacksteder passed away in November 2007 
below, together with Lie's theorem that every connected Lie group is locally linear. ${ }^{1}$. We remark it follows from the definition that a complex Lie group is semi-algebraic if and only if its radical is semi-algebraic.

Corollary 1.1. A connected complex Lie group whose exponential map is surjective is semi-algebraic.

If $G$ is exponential, the functorial properties of the exponential map (cf. the beginning of the proof of Proposition 4.1 below) imply that every quotient group is also exponential. In particular, if $G$ is exponential the adjoint group is also. As was shown in [12] (see Corollary 4) if $Z(G)$ is connected and $\operatorname{Ad}(G)$ is exponential, so is $G$. Hence, what remains to be proved here is that if a complex connected Lie group is exponential its center is connected. We note that in the solvable case this is actually true for any real group (see Wüstner [21]).

The proof of Theorem 1 is given in the last section. The results obtained in the intervening sections are needed to show that the proof for a general complex Lie group can be reduced to proving the theorem for a group satisfying certain restrictive conditions.

\section{Semi-algebraic Lie algebras}

If $\mathfrak{g}$ is a complex Lie Algebra, $\mathfrak{z}(\mathfrak{g})$ will denote its center, $\mathfrak{r}(\mathfrak{g})$ its radical, and $\mathfrak{s}(\mathfrak{g})$ a Levi factor. A Lie algebra $\mathfrak{g}$ will be called semi-algebraic if it has the properties:

(1) $\mathfrak{r}(\mathfrak{g})=\mathfrak{n} \oplus \mathfrak{a}$ is a semidirect sum, where $\mathfrak{a}$ is abelian and $\mathfrak{n}$ is a nilpotent ideal.

(2) The action of $\mathfrak{a}$ on $\mathfrak{n}$ is semisimple.

(3) $[\mathfrak{s}, \mathfrak{a}]=0$.

Note that this definition does not generally determine a unique choice of $\mathfrak{a}$. For instance, if $\mathfrak{r}=\mathfrak{g}$ is solvable and has a nontrivial center $\mathfrak{z}(\mathfrak{g}) . \mathfrak{n}$ might be the nilradical which would then contain $\mathfrak{z}(\mathfrak{g})$, but it could be a smaller ideal and then part of $\mathfrak{z}(\mathfrak{g})$ would then be included in $\mathfrak{a}$. However, if $\mathfrak{z}(\mathfrak{g})=0, \mathfrak{n}$ must be the nilradical (the largest nilpotent ideal of $\mathfrak{g}$ ). It is known that Lie algebras of algebraic groups are semi-algebraic (see Section 5 below).

An example that illustrates some of the arguments that will be made below is given by the complex solvable Lie algebra $\mathfrak{r}$ generated by $\left\{e_{1}, e_{2}, e_{3}, e_{4}\right\}$ with the following relations: $\left[e_{1}, e_{2}\right]=e_{3},\left[e_{1}, e_{4}\right]=e_{4}$. One can verify that $\mathfrak{r}$ is not semi-algebraic, but that dividing by the center $\mathfrak{z}(\mathfrak{r})=\mathbb{C} e_{3}$ produces a semi-algebraic algebra $\mathfrak{r}_{1}$ and dividing $\mathfrak{r}_{1}$ by its center produces a semi-algebraic Lie algebra with a trivial center. However, this process of successive divisions by the center does not always lead to a semi-algebraic Lie algebra as will be shown by an example in Section 5 below.

We introduce some notation by recalling the following classical result:

Lemma 2.1. Let $\psi: \mathfrak{a} \longrightarrow \operatorname{End}(V)$ be a representation of the complex abelian Lie algebra $\mathfrak{a}$ on a complex vector space $V$ and suppose that for every $\alpha \in \mathfrak{a}, \psi(\alpha)$ is semisimple. Then there is a basis $\mathcal{F}=\left\{f_{1}, \ldots, f_{n}\right\}$ of $V$ such that every $f_{j}$ is a simultaneous eigenvector (with eigenvalue $\lambda_{j}(\alpha)$ ) of $\psi(\alpha)$ for all $\alpha \in \mathfrak{a}$.

\footnotetext{
${ }^{1}$ The referee has pointed out that the notion of a semi-algebraic complex Lie group is actually equivalent to that of a split Lie group (see [20] or [1])
} 
Lemma 2.1 implies that if $\mathfrak{r}$ is a complex solvable semi-algebraic Lie algebra there are linear functionals on $\mathfrak{a},\left\{\lambda_{0}=0, \lambda_{1}, \ldots \lambda_{s}\right\}$ and corresponding subspaces of $\mathfrak{n}$, $\left\{\mathfrak{n}\left(\lambda_{0}\right)=\mathfrak{z}(\mathfrak{n}), \mathfrak{n}\left(\lambda_{1}\right), \ldots, \mathfrak{n}\left(\lambda_{s}\right)\right\}$ such that $\mathfrak{n}$ is a direct sum $\mathfrak{n}=\bigoplus_{j=0}^{s} \mathfrak{n}\left(\lambda_{j}\right)$ and $[\alpha, x]=$ $\lambda_{j}(\alpha) x$ for $\alpha \in \mathfrak{a}, x \in \mathfrak{n}\left(\lambda_{j}\right)$. As usual, one has $\left[\mathfrak{n}\left(\lambda_{j}\right), \mathfrak{n}\left(\lambda_{k}\right)\right] \subseteq \mathfrak{n}\left(\lambda_{j}+\lambda_{k}\right)$. It is obvious that there is a basis $\mathcal{F}=\left\{f_{1}, \ldots, f_{n}\right\}$ for $\mathfrak{n}$ with the property that every $f_{i} \in \mathfrak{n}\left(\lambda_{j}\right)$ for some $j=j(i)$.

Lemma 2.2. Suppose that $\mathfrak{r}=\mathfrak{n} \oplus \mathfrak{a}$ is a complex semi-algebraic solvable Lie algebra and that $\mathfrak{n}=\mathfrak{n}^{1} \supset \mathfrak{n}^{2} \supset \cdots \supset \mathfrak{n}^{p}=\{0\}$ is a sequence of characteristic ideals of $\mathfrak{n}$. Then there is a basis $\mathcal{B}=\left\{e_{1}, \ldots, e_{n}\right\}$ for $\mathfrak{n}$ which has the property of the basis $\mathcal{F}$ and in addition for every $\mathfrak{n}^{j}, \mathcal{B}^{j}=\mathcal{B} \cap \mathfrak{n}^{j}$ spans $\mathfrak{n}^{j}$.

Proof. First note that $\mathfrak{n}$ is a (not necessarily direct) sum of eigenvectors, that is, of the elements of the spaces $\mathfrak{n}\left(\lambda_{j}\right)$ and each non-trivial eigenvector is a minimal invariant subspace. This then implies that the representation $\psi$ as in Lemma 2.1 is completely reducible, cf. Theorem 11.20 in [18]. It then follows that the restriction of $\psi$ to any invariant subspace is also completely reducible, cf. Proposition 11.19 in [18]. In particular, the restriction of $\psi$ to every $\mathfrak{n}^{j}$ is completely reducible.

Let $D$ be a semisimple derivation of a nilpotent Lie algebra $\mathfrak{n}$ and let $\mathfrak{n}=\mathfrak{n}^{1} \supset$ $\ldots \supset\{0\}$ be the lower (or descending) central series. By Lemma 2.2 (taking $\mathfrak{a}=\mathbb{C}$ ), the basis $\mathcal{B}$ for $\mathfrak{n}$ consists of eigenvectors of $D$ and the elements of $\mathcal{B}_{p}=\mathcal{B} \cap\left(\mathfrak{n}^{p} \backslash \mathfrak{n}^{p+1}\right)$ are linearly independent.

Lemma 2.3. Let $\mathfrak{r}$ be as in Lemma 2.2 with $\mathfrak{a}=\mathbb{C}$ related to a derivation $D$ as above. If $D e_{i}=0$ for all $e_{i} \in \mathcal{B}_{1}=\mathcal{B} \cap\left(\mathfrak{n}^{1} \backslash \mathfrak{n}^{2}\right)$, then $D \equiv 0$.

Proof. If $D \not \equiv 0$, let $p>1$ be the smallest integer such that $D e_{i}=\lambda e_{i} \neq 0$ for some $e_{i} \in \mathcal{B}_{p}$. Then $e_{i}=\sum c_{j k}\left[e_{j}, e_{k}\right]$, where the summation is over $e_{j} \in \mathfrak{n}^{p-1}$ and $e_{k} \in \mathfrak{n}^{1}$. But by definition of $p, D e_{j}=0 \bmod \left(\mathfrak{n}^{p}\right)$ and $D e_{k}=0 \bmod \left(\mathfrak{n}^{2}\right)$ and therefore $D e_{i}=\lambda e_{i}=\sum c_{j k}\left(\left[D e_{j}, e_{k}\right]+\left[e_{j}, D e_{k}\right]\right)=0$ by $[8$, Proposition 5, p.25], because each term in the sum is $0 \bmod \left(\mathfrak{n}^{p}\right)$. This contradiction proves the lemma.

\section{Exptrivial Endomorphisms}

If $V$ is a finite dimensional complex vector space, a linear map $L: V \longrightarrow V$ will be called exptrivial if $\operatorname{Exp}_{V}(L)=I_{V}$, where $I_{V}$ is the identity map of $V$ and $\operatorname{Exp}_{V}$ is defined by power series. It is obvious that if $L$ is semisimple then it is exptrivial if and only if all of its eigenvalues are integer multiples of $2 \pi i$.

Proposition 3.1. Let $A$ and $N$ be nilpotent endomorphisms of a vector space $V$ and suppose $\operatorname{Exp}_{V}(A)=\operatorname{Exp}_{V}(N)$. Then $A=N$.

Proof. This is because for every integer $n, \operatorname{Exp}_{V}(n A)=\operatorname{Exp}_{V}(A)^{n}=\operatorname{Exp}_{V}(N)^{n}=$ $\operatorname{Exp}_{V}(n N)$. Thus the polynomial $p(t)=\operatorname{Exp}_{V}(t A)-\operatorname{Exp}_{V}(t N)$ has infinitely many distinct zeros and so must be identically zero. Taking the derivative at $t=0$ yields $A=N$.

Proposition 3.2. Let $A$ and $B$ be linear maps of $V$ such that $A$ is nilpotent and $\operatorname{Exp}_{V}(A)=\operatorname{Exp}_{V}(B)$. Suppose that $B=S+N$, where is $S$ semisimple and $N$ is nilpotent. Then $S$ is exptrivial and $A=N$. Hence $A, B, S$, and $N$ commute pairwise. 
Proof. Applying the uniqueness of the multiplicative Jordan decomposition [7, p.9596] to $\operatorname{Exp}_{V}(A)=I_{V} \operatorname{Exp}_{V}(A)=\operatorname{Exp}_{V}(B)=\operatorname{Exp}_{V}(S) \operatorname{Exp}_{V}(N)$ gives $\operatorname{Exp}_{V}(S)=$ $I_{V}$ and $\operatorname{Exp}_{V}(A)=\operatorname{Exp}_{V}(N)$. But since $A$ and $N$ are nilpotent, $A=N$ by Proposition 3.1.

Corollary 3.1. A linear map $L: V \longrightarrow V$ is exptrivial if and only if it is semisimple and each of its eigenvalues is an integer multiple of $2 \pi i$.

Proof. The "if" part is obvious. Applying Proposition 3.2 with $A=0, B=L$, shows that $N=A=0$. Thus $L$ is semisimple and therefore $I_{V}=\operatorname{Exp}_{V}(L)$ implies that all of its eigenvalues are integer multiples of $2 \pi i$.

Proposition 3.3. Let $G$ be a complex connected Lie group with Lie algebra $\mathfrak{g}$, adjoint group $\operatorname{Ad}(G)$, and projection $\pi_{A}: G \longrightarrow \operatorname{Ad}(G)$. If $z=\exp _{G}(\sigma) \in Z(G)$ then ad $d_{\sigma}$ is exptrivial. Conversely, if ad $d_{\sigma}$ is exptrivial, $\exp _{G}(\sigma) \in Z(G)$.

Proof. Note that $\pi_{A}(Z(G))=1$ and $A_{G}\left(\exp _{G}(\sigma)\right)=\operatorname{Exp}_{V}\left(a d_{\sigma}\right)$ (where $V=\mathfrak{g}$ ). If $z=\exp _{G}(\sigma) \in Z(G), A_{G}(z)-I_{V}=\operatorname{Exp}_{V}\left(a d_{\sigma}\right)-I_{V}=0$, since $Z(G)=\operatorname{Ker}\left(A d_{G}\right)$ $\left[19\right.$, p.116]. Hence $a d_{\sigma}$ is exptrivial by Corollary 3.1.

For the converse, suppose that $a d_{\sigma}$ is exptrivial and set $z=\exp _{G}(\sigma)$. Then $A d_{G}(z)=\operatorname{Exp}_{V}\left(a d_{\sigma}\right)=I_{V}$ by Corollary 3.1. Hence $z \in \operatorname{Ker}\left(A d_{G}\right)=Z(G)$.

Sometimes the assumption that $z \in Z(G)$ in Proposition 3.3 is redundant. For example if $G$ is connected and semisimple, $Z(G) \subseteq E(G)$, even if $G$ is not exponential. In fact, let $K$ be a maximal compact subgroup of $G$. Then because we are in a complex semisimple group $Z(G)$ is finite (see [6]). Hence the group $Z(G) \cdot K$ is compact. It contains $K$ and therefore by maximality $Z(G) \cdot K=K$, so $Z(G) \subseteq K$. Since $K$ is connected and hence exponential $Z(G) \subseteq E(G)$. We note that our simple argument of this fact in the complex semisimple case actually holds more generally. Wüstner has shown $Z(G) \subseteq E(G)$ in any connected Lie group.

\section{Semi-algebraic Groups}

A connected Lie group $G$ will also be called semi-algebraic if its Lie algebra is semi-algebraic.

Proposition 4.1. Let $G$ be a complex connected Lie group with center $Z(G)$ and denote the identity component of $Z(G)$ by $Z_{1}(G)$. If $H=G / Z_{1}(G)$, and $\pi: G \longrightarrow H$, $\pi^{*}: \mathfrak{g} \longrightarrow \mathfrak{h}$ are the natural maps, then:

(1) If $J \subseteq G$ is a closed normal subgroup and $G$ is exponential, $G / J$ is also exponential.

(2) $G$ is exponential if and only if $H$ is.

(3) If $E(G) \supseteq Z(G)$ and $E(H) \supseteq Z(H)$, then $Z(G)$ is connected if and only if $Z(H)$ is connected.

(4) If the Lie algebra $\mathfrak{g}$ of $G$ is semi-algebraic and the Lie algebra of $J$ is a characteristic ideal of $\mathfrak{g}$, the Lie algebra of $G / J$ is semi-algebraic. In particular, the Lie algebra of $H$ is semi-algebraic,

Proof. The relation $\exp _{H} \circ \pi^{*}=\pi \circ \exp _{G}[19$, Theorem 3.32] implies that if $G$ is exponential $H$ and $G / J$ are also. To prove the converse for $H$, note that if $h=$ $\exp _{H}(\eta)=\exp _{H}\left(\pi^{*} \gamma\right)$, any element of the coset $h=\exp _{G}(\gamma) Z_{1}(G)$ is of the form 
$\exp _{G}(\gamma) \exp _{G}(\sigma)=\exp _{G}(\gamma \sigma)$ for $\sigma \in Z_{1}(G)$. That is, any element of any coset is in the range of $\exp _{G}$. To prove the third assertion, first note that if $\gamma, \alpha \in \mathfrak{g}$ and $[\gamma, \alpha]=\lambda \alpha$ with $\lambda \neq 0$ and $\alpha \neq 0$, then $\pi^{*}[\gamma, \alpha]=\left[\pi^{*} \gamma, \pi^{*} \alpha\right]=\lambda \pi^{*} \alpha$ with $\pi^{*} \alpha \neq 0$ (since $\operatorname{Ker}\left(\pi^{*}\right)=\mathfrak{z}(\mathfrak{g})$ is the Lie algebra of $Z_{1}(G)$ ). Thus the non-zero eigenvalues of $a d_{\gamma}$ and $a d_{\pi^{*} \gamma}$ are the same, although their multiplicities will be reduced by the dimension of $Z_{1}(G)$. Therefore, Proposition 3.3 shows that $\gamma \neq 0$ is exptrivial if and only if $\pi^{*} \gamma \neq 0$ is exptrivial. If $Z(H) \subseteq E(H)$ is not connected there is an exptrivial $\pi^{*} \gamma$ such that $\exp _{H}\left(\pi^{*} \gamma\right) \in Z(H) \backslash Z_{1}(H)$. In particular, $\exp _{H}\left(\pi^{*} \gamma\right) \neq 1_{H}$, so $\exp _{G}(\gamma) \in Z(G) \backslash Z_{1}(G)$ and $Z(G)$ is not connected. Conversely, if $Z(G) \subseteq E(G)$ is not connected there is an exptrivial $\gamma \in \mathfrak{g}$ such that $\exp _{G}(\gamma) \in Z(G) \backslash Z_{1}(G)$. It will be shown that $\exp _{H}\left(\pi^{*} \gamma\right) \notin Z_{1}(H)$, hence $Z(H)$ is not connected. To see this, first note that every element of $Z_{1}(H)$ lies on a one-parameter subgroup of $Z_{1}(H)$ and any such subgroup has a preimage in the identity component of $\pi^{-1}\left(Z_{1}(H)\right)$. The Lie algebra of this component is $\left(\pi^{*}\right)^{-1} \mathfrak{z}(\mathfrak{h})=\mathcal{C}_{2}$ (the second term in the upper central series for $\mathfrak{g}$ ). This Lie algebra is nilpotent and consequently contains no exptrivial element other than 0 . Then $\pi^{*} \gamma$ has a preimage there that is not exptrivial, so $\pi^{*} \gamma$ is not exptrivial. But this is impossible, since $\gamma$ is exptrivial. This contradiction shows that $Z(H)$ is not connected. The fourth assertion is an easy consequence of Lemma 2.2 .

The construction of $H$ from $G$ can be iterated. That is, one can define the sequence of groups and maps $G=G_{0}, G_{1}=G / Z_{1}\left(G_{0}\right), \ldots, G_{p+1}=G_{p} / Z_{1}\left(G_{p}\right), \pi_{p}: G_{p-1} \longrightarrow$ $G_{p}$ the natural map, and $\phi_{p}=\pi_{p} \circ \pi_{p-1} \circ \cdots \circ \pi_{1}$. Note that for large $p, G_{p}=G_{p+1}=$ $G_{*}, \phi_{p}=\phi_{p+1}=\phi$ and the center of $G_{*}$ is discrete. A sequence of subgroups of $G$, that are easily verified to be closed, connected, normal, and nilpotent

$$
N_{1}=Z_{1}(G) \subseteq N_{2} \subseteq N_{3} \subseteq \ldots N_{*}
$$

is defined by $N_{p+1}=\phi_{p}^{-1}\left(Z_{1}\left(G_{p}\right)\right)(p>0)$. Here $N_{*}=\phi_{p}^{-1}\left(Z_{1}\left(G_{*}\right)\right)$ for large $p$ is a connected, normal, nilpotent subgroup of $G$ and $\phi(G)=G_{*}=G / N_{*}$. Then the properties of $G$ and $G_{*}$ are related just as those of $G$ and $H$ were in that results stated above. That is, Proposition 4.1 is correct if $H$ is replaced by $G_{*}$ and $\pi$ is replaced by $\phi$. These remarks give:

Corollary 4.1. To every complex connected Lie group $G$ there is associated a connected nilpotent normal subgroup $N_{*}$ with the properties:

(1) $G$ is exponential if and only if $G_{*}=G / N_{*}$ is exponential.

(2) If $G$ and $G_{*}$ are exponential then $Z\left(G_{*}\right)$ is connected if and only if $Z(G)$ is connected.

(3) If the Lie algebra of $G$ is semi-algebraic, so is the Lie algebra of $G_{*}$.

(4) The adjoint representation of $\mathfrak{g}_{*}$ (the Lie algebra of $G_{*}$ ) is faithful.

(5) $Z\left(G_{*}\right)$ is discrete.

It will sometimes be convenient in what follows to assume that $Z(G)$ is discrete, that is to replace $G$ by $G_{*}$.

Proposition 4.1 gives an extension of a result of Dixmier [4] from complex solvable groups to arbitrary complex Lie groups. 
Corollary 4.2. If a simply connected complex Lie group is exponential it must be nilpotent.

Proof. Since $G$ is exponential, by Proposition 4.1 so is $G / \operatorname{Rad}(G)$. On the other hand since $G$ is simply connected it is a semidirect product $G=R \cdot S$, where $S$ is a Levi factor. Hence each of the factors is also simply connected and $G / R=S$. However, no non-trivial simply connected semisimple group can be exponential [20]. This means $G / \operatorname{Rad}(G)$ is trivial and $G$ is solvable. But by [4], a solvable, simply connected, complex Lie group $G$ is nilpotent.

\section{Exponential Linear Groups}

In a sequence of groups and maps $G=G_{0} \longrightarrow G_{1} \longrightarrow \ldots \longrightarrow G_{p}=G_{*}$ as described above it can happen (even if the Lie algebra of $G$ is not semi-algebraic) that beyond some step in the sequence the Lie algebras are semi-algebraic. In this case, the Lie algebra of $G$ is said to be residually semi-algebraic.

Before stating the next proposition we recall some known facts about complex algebraic groups (see [16] or [7, Ch. VII]). Let $G$ be a connected complex linear algebraic group $G=N \ltimes P$ (semi-direct product) be its algebraic group Levi decomposition, where $N$ is the unipotent radical of $G$ and $P$ is a reductive subgroup. Because $P$ is reductive, $P=A \cdot[P, P]$, where $A$ is a complex torus and $[P, P]=S$, the derived group of $P$, is semisimple. Since $N$ is normal in $G, N A$ is an algebraic subgroup, of $G$ and because $A$ is centralized by $P, N A$ is normal in $G$. Moreover, because $N$ and $A$ are solvable and connected so is $N A$. Finally,

$$
G / N A=P / A=S / A \cap S,
$$

and so $G / N A$ is semisimple. Thus $N A=\operatorname{Rad}(G)$, the radical of $G$. Moreover, it is easy to see from the above that $N A \cap S=\left\{1_{G}\right\}$, so that $G$ is a semi-direct product of $\operatorname{Rad}(G)$ with $S$ and $S$ is a Levi factor. Thus in the case of complex algebraic groups the complex Lie group Levi decomposition is actually a semidirect product,

$$
G=\operatorname{Rad}(G) \ltimes S,
$$

as is the radical,

$$
\operatorname{Rad}(G)=N \ltimes A .
$$

It was noted in Section 2 that the Lie algebras of algebraic groups are semi-algebraic. This follows from results in [16]. The Lie algebras of $S, A$, and $N$ can be taken as the algebras $\mathfrak{s}, \mathfrak{a}$, and $\mathfrak{n}$ in the definition of semi-algebraic. This result could be used to shorten the proof of Proposition 5.1 slightly. The main argument needed for the result appears in the last paragraph of the proof of Proposition 5.1.

Proposition 5.1. Let $G \subseteq \mathrm{GL}(n, \mathbb{C})$ be a complex connected linear Lie group. If $G$ is exponential then its Lie algebra is semi-algebraic. 
Proof. Let $G^{\sharp}$ be the algebraic hull of $G$. So that $G \subseteq G^{\sharp} \subseteq \mathrm{GL}(n, \mathbb{C})=\mathrm{GL}(V)$. By the remarks above, the algebraic group $G^{\sharp}=N A S$, where $N$ is the unipotent radical, $A$ is a complex torus and $S$ is a complex semisimple Lie group, and the subgroup $N A$ is the radical of $G^{\sharp}$. The hull of a connected solvable group is solvable (by Lie's theorem), the hull of a normal subgroup is normal in the larger hull (see [2, p. 57 d]) and finally the hull of something connected is again connected (see [2, p. 55]). Let $R$ be the radical of $G$. Then $G^{\sharp}=(R S)^{\sharp}=R^{\sharp} S^{\sharp}$ (See [7, Corollary p. 54]). But $S^{\sharp}=S$, because complex semisimple groups are algebraic (over $\mathbb{Q}$ ). Since $R^{\sharp}$ is solvable, normal and connected and $G^{\sharp}=R^{\sharp} S$, we see that $R^{\sharp}=\operatorname{Rad}\left(G^{\sharp}\right)$.

We now show that $R \cap N$ is connected. Let $1_{G} \neq g \in G \cap N$. Since $G$ is exponential $g=\exp _{G}(x)$, for some $x \in \mathfrak{g}$. But also, because $g \in N, g=\exp _{N}(y)$, where $y$ is nilpotent. Hence $\exp _{G}(k x)=\exp _{N}(k y)$ for all integers $k$ so $\exp _{G} k x \in N$ for all $k$. It follows that $\exp _{G}(t x) \in N$ for all $t \in \mathbb{R}$. Thus $x \in \mathfrak{n}$ and since $x \in \mathfrak{g}, x \in \mathfrak{n} \cap \mathfrak{g}$, the Lie algebra of $N \cap G$. This means $\exp _{G}(t x) \in N \cap G$ for all $t$ and shows $N \cap G$ is exponential and therefore connected. Now $N \cap G$ is a normal nilpotent subgroup of $G$ because $N$ is normal in $G^{\sharp}$. Since $N \cap G$ is connected it is contained in $R$ and so coincides with $R \cap N$. Therefore $R \cap N$ is connected.

The connected nilpotent subgroup $R \cap N$ is Zariski closed in $\mathrm{GL}(V)$. Hence it's also Euclidean closed in GL(V) and so in $R$. It's also normal in $R$ since $N$ is normal in $R^{\sharp}$ being the unipotent radical. Now $R / R \cap N \subseteq R N / N \subseteq R^{\sharp} / N=A$ so $R / R \cap N$ is a connected (because $R$ is connected) subgroup of a diagonalizable connected group. Hence it consists of semisimple elements and therefore $R / R \cap N$ is itself a connected diagonalizable group (split torus). Moreover, since $R \cap N$ is simply connected (since it's unipotent) we conclude from Prop 4.41 (p.129) of [11] that $R=(R \cap N) \ltimes B$ (semi-direct product), where $B$ is a diagonalizable connected subgroup of $R$. Hence (again using the normality of $R \cap N$ we see $R^{\sharp}=(R \cap N)^{\sharp} \ltimes B^{\sharp}$. But the first factor is contained in $N$ and the second is diagonalizable (as the hull of such a group). Since $R^{\sharp}$ is the semidirect product of $N$ and $A$ it follows that $(R \cap N)^{\sharp}=N$ and since $R \cap N$ is also Zariski closed it must be $N$.

Let $\mathfrak{a}, \mathfrak{b}, \mathfrak{n}, \mathfrak{r}$ and $\mathfrak{s}$ be the Lie algebras of the connected complex Lie groups $A, B$, $N, R$ and $S$. As $R=N \ltimes B$ (semi direct product) it follows that $\mathfrak{r}=\mathfrak{n} \oplus \mathfrak{b}$ (semi-direct sum). Because $\mathfrak{n}$ is a nilpotent ideal and $\mathfrak{b}$ is an abelian subalgebra of $\mathfrak{r}$ this proves that condition (1) of the definition of semi-algebraic is satisfied.

To verify that conditions (2) and (3) of the definition are satisfied, note first that $A$ and therefore also the abelian algebra $\mathfrak{a}$ acts completely reducibly on $V$. Hence the same is true of the subalgebra $\mathfrak{b}$. As is well known [1, I, §5.4, Lemma 2] and easy to see, if the matrix units in $\operatorname{End}(V)$ are denoted by $e_{i, j}$ and $d=\sum_{i=1}^{n} \lambda_{i, i} e_{i, i}$ is a diagonal element then $\left[d, e_{k, l}\right]=\left(\lambda_{k, k}-\lambda_{l, l}\right) e_{k, l}$. Hence $\operatorname{ad}_{d}$ is diagonal on $\operatorname{End}(V)$. Since $\mathfrak{b}$ lies in the subalgebra $\mathfrak{r}$ and the latter is an $a d_{b}$ invariant subspace for every $b \in \mathfrak{b}$ we see $\mathfrak{b}$ acts completely reducibly on $\mathfrak{r}$. Moreover, $\mathfrak{n}$ is an ideal in $\mathfrak{r}$ so $\mathfrak{b}$ acts completely reducibly on $\mathfrak{n}$. This proves (2).

Finally since $A$ and $S$ commute in $G^{\sharp}$ we get $[\mathfrak{a}, \mathfrak{s}]=(0)$. Hence also $[\mathfrak{b}, \mathfrak{s}]=(0)$, proving (3).

Corollary 5.1. Let $G$ be any complex connected Lie group. If $G$ is exponential, then its Lie algebra is residually semi-algebraic. 
Proof. Since $G$ is exponential so is the complex connected linear Lie group $\operatorname{Ad}(G) \subseteq$ $\operatorname{GL}(\mathfrak{g})$. Hence by Proposition 5.1 the Lie algebra of $\operatorname{Ad}(G)$ is semi-algebraic. Since $Z(G)=\operatorname{Ker}(\operatorname{Ad}(G))$, the Lie algebra of $G$ itself is residually semi-algebraic.

The hypothesis that $G$ is exponential is essential in Proposition 5.1, that is, there are examples of complex linear groups that are not even residually semi-algebraic. Let $\mathfrak{r}$ be the complex solvable Lie algebra generated by $\left\{e_{1}, e_{2}, e_{3}\right\}$ with the following relations: $\left[e_{1}, e_{2}\right]=e_{2}+e_{3},\left[e_{1}, e_{3}\right]=e_{3},\left[e_{2}, e_{3}\right]=0$. Then it can verified that $\mathfrak{r}$ is a semidirect sum $V \oplus U$, where $e_{1}$ spans $U$ and $V$ consists of linear combinations of $e_{2}$ and $e_{3}$. Moreover, $\mathfrak{z}(\mathfrak{r})$ is trivial and the action of $U$ on $V$ is not semisimple, so $\mathfrak{r}$ is not semi-algebraic or even residually semi-algebraic. In fact, the only connected group having that Lie algebra is non-exponential and not residually semi-algebraic, but since that group has a trivial center, its adjoint representation is faithful.

The connected complex group $R$ having $\mathfrak{r}$ as its Lie algebra can be parameterized by $(x, y, z)$ and a faithful representation is given by:

$$
\psi(x, y, z)=\left(\begin{array}{ccc}
e^{z} & z e^{z} & x \\
0 & e^{z} & y \\
0 & 0 & 1
\end{array}\right)
$$

One can verify that $Z(R)$ is trivial and $R$ is simply connected. The fact that $R$ is not exponential follows from Proposition 5.1 but can also be verified directly, for instance by showing that the element $\psi(0, y, 2 \pi i)$ is not in the range of $\exp _{R}$.

Our goal in this paper is to prove that if a complex connected Lie group $G$ is exponential then its center $Z(G)$ is connected. Corollaries 4.1 and 5.1 show that it suffices to carry out the proof for groups with semi-algebraic Lie algebras and discrete centers.

\section{The Semisimple Case}

Lai [9] and Wüstner [20] have proved that a complex semisimple Lie group $S$ is exponential only if its center is trivial. A slight variation on an argument used in their proofs will be needed in our treatment of the general case where $G$ is a connected complex exponential Lie group. The argument uses the next statement, which follows from a result in [17, p. 112]: If $S$ is a semisimple Lie group with Lie algebra $\mathfrak{s}$ there is a nilpotent element $\dot{w} \in \mathfrak{s}$ such that if $[y, \dot{w}]=0$ then $y$ is nilpotent. In particular, if $a d_{y}$ is exptrivial and $[y, \dot{w}]=0$, then $y=0$

Here it will be convenient to use the following version of the Lai-Wüstner result:

Lemma 6.1. Let $S$ be a semisimple Lie group with Lie algebra $\mathfrak{s}$, let $\dot{w}$ be as above, and $z=\exp _{S}(\dot{z}) \in Z(G)$. If for some for some $\dot{s} \in \mathfrak{s}$, $\exp _{S}(\dot{s})=z \exp _{S}(\dot{w})$, then $z=1_{S}$ and $\dot{s}=\dot{w}$.

Proof. It can be supposed that $S \subseteq G L(n, \mathbb{C})$ by [6, Theorem 3.2, XVII.4] and $\mathfrak{s} \subseteq$ $g l(n, \mathbb{C})$ is splittable. This implies that $\dot{s}=\dot{x}+\dot{y} \in g l(n, \mathbb{C})$, where $\dot{x}$ is nilpotent, $\dot{y}$ is semisimple, and $[\dot{x}, \dot{y}]=0$. Then by the uniqueness of the multiplicative Jordan decomposition $\operatorname{Exp}(\dot{x})=\exp _{S}(\dot{x})=\operatorname{Exp}(\dot{w})=\exp _{S}(\dot{w})$ and $\operatorname{Exp}(\dot{y})=\exp _{S}(\dot{y})=z$. But by Proposition $3.2, \dot{x}=\dot{w}$, so $[\dot{y}, \dot{w}]=0$. Then by the property of $\dot{w}$, this implies that $\dot{y}=0$, hence $z=1_{S}$ and $\dot{s}=\dot{x}=\dot{w}$. 


\section{Semidirect Products}

The following proposition is proved in [1, VII, §2, Proposition 10].

Proposition 7.1. Let $\mathfrak{g}$ be a Lie algebra, $\mathfrak{a}$ a commutative subalgebra, and $\mathfrak{c}$ the centralizer of $\mathfrak{a}$ in $\mathfrak{g}$. Suppose that for every $a \in \mathfrak{a}$, ad $d_{a} \in \operatorname{End}(\mathfrak{g})$ is semisimple. Then $\mathfrak{h} \subseteq \mathfrak{g}$ is a Cartan subalgebra of $\mathfrak{c}$ if and only if it is a Cartan subalgebra of $\mathfrak{g}$ containing $\mathfrak{a}$.

The case where $\mathfrak{a}$ is a 1-dimensional Lie algebra spanned by a single ad-semisimple element gives:

Corollary 7.1. If $a \in \mathfrak{g}$ and $a d_{a}$ is semisimple, there is a Cartan subalgebra $\mathfrak{h}$ of $\mathfrak{g}$ containing a.

Some solvable Lie algebras that will arise in proofs below will now be described along with explicit formulas for the multiplication and the exponential map in the corresponding simply connected groups (cf. [13, Section 3]).

The following assumptions and notation will be in effect for the remainder of this section.

$U \cong \mathbb{C}^{n}$ and $V \cong \mathbb{C}^{m}$ will be complex vector spaces regarded as abelian Lie algebras and the corresponding simply connected Lie groups will be denoted by $\mathcal{U}$ and $\mathcal{V}$. The exponential map $\exp _{\mathcal{V}}: V \longrightarrow \mathcal{V}$ is both a diffeomorphism and an isomorphism of abelian groups. If $v \in \mathcal{V}$, the corresponding element of $V$ will be denoted by $\dot{v}$ and similarly for $u \in \mathcal{U}$. If $A \in \operatorname{End}(V), \operatorname{Exp}_{V}(A)$ will denote the endomorphism defined by the exponential power series. Notation will be abused slightly here by identifying endomorphisms of $V$ and $\mathcal{V}$ that are conjugate by $\exp _{\mathcal{V}}$; for instance, by writing $A\langle v\rangle$ rather than $\exp _{v} \circ A \circ \exp _{v}^{-1}\langle v\rangle$ for the endomorphism of $\mathcal{V}$ corresponding to $A \in \operatorname{End}(V)$. Suppose that $\phi: U \longrightarrow \operatorname{End}(V)=\operatorname{Der}(V)$ is a morphism of Lie algebras. Then $\phi$ defines the structure of a semidirect sum $\mathfrak{h}=V \oplus_{\phi} U$ by the relation $[\dot{u}, \dot{v}]=\phi(\dot{u})<\dot{v}>$.

Lemma 7.1. Let $\Phi: \mathcal{U} \longrightarrow \operatorname{Aut}(\mathcal{V})$ be defined by $\Phi(u)\langle v\rangle=\operatorname{Exp}_{V}(\phi(\dot{u}))<v>$. The multiplication law in the simply connected group $\mathcal{H}=\mathcal{V} \ltimes_{\Phi} \mathcal{U}$ is then

$$
\left(v_{1}, u_{1}\right)\left(v_{2}, u_{2}\right)=\left(v_{1}+\operatorname{Exp}_{V}\left(\phi\left(\dot{u}_{1}\right)\right)<v_{2}>, u_{1}+u_{2}\right) .
$$

The one-parameter subgroup of $\mathcal{H}$ determined by $\dot{z}=(\dot{v}, \dot{u})$ is

$$
z(t)=\left(\int_{0}^{t} \operatorname{Exp}_{V}(s \phi(\dot{u}))<\dot{v}>d s, t \dot{u}\right) .
$$

The Lie algebra of $\mathcal{H}$ is $\mathfrak{h}$ as defined above.

Proof. It is clear that the formula for $\Phi$ defines a representation of $\mathcal{U}$ as a group of automorphisms of $\mathcal{V}$ and that (1) defines the corresponding group multiplication. That eq. (2) defines a 1-parameter subgroup then follows from the calculus formula

$$
\int_{0}^{t+u} \operatorname{Exp}_{V}(s A) d s=\int_{0}^{t} \operatorname{Exp}_{V}(s A) d s+\operatorname{Exp}_{V}(t A) \int_{0}^{u} \operatorname{Exp}_{V}(s A) d s,
$$


with $A$ a vector space endomorphism and the multiplication law (1) for $\mathcal{H}$. Differentiating eq. (2) at $t=0$ establishes a correspondence between $\mathfrak{h}$ and the Lie algebra of $\mathcal{H}$. To verify that the bracket operations in the algebras agree one can, for instance, compute $A d_{z(t)}=\operatorname{Exp}_{V}\left(\operatorname{tad}_{\dot{z}}\right)$ using eq. (1) and differentiate at $t=0$.

\section{Corollary 7.2.}

(1) Suppose that $\phi(\dot{u})$ as defined just before the statement of Lemma 7.1 is semisimple for every $\dot{u} \in U$. Then $\mathfrak{h}=V \oplus_{\phi} U$ is semi-algebraic. In this case, $V=\bigoplus_{0}^{N} V_{j}$ is a direct sum of subspaces that are invariant under $\phi(\dot{u})$ for every $\dot{u} \in U$ and there are elements $\lambda_{0}, \ldots, \lambda_{N}$ in $U^{*}$ such that for $\dot{v}_{j} \in V_{j}$, $\phi(\dot{u})<\dot{v}_{j}>=\lambda_{j}(\dot{u}) \dot{v}_{j}$.

(2) Suppose in addition, $\dot{u} \in U$ is exptrivial so that $\lambda_{j}(\dot{u})=2 \pi i n_{j}$ with $n_{j} \in \mathbb{Z}$ for every $j$. If $\dot{v}=\sum \dot{v}_{j}$ with $\dot{v}_{j} \in V_{j}$, then $\exp _{\mathcal{H}}((\dot{v}, \dot{u}))=(w, u)$, where $w_{j}=v_{j}$ if $n_{j}=0$ and $w_{j}=0$ if $n_{j} \neq 0$.

Proof. The first assertion is clear from the definition of semi-algebraic and Lemma 2.1. The last follows by applying Lemma 7.1 and calculating eq. (2) with $t=1$.

Corollary 7.3. Assume as in Corollary 7.2 that $\phi(\dot{u})$ is semisimple. If in the notation of Lemma $7.1(v, u) \in Z(\mathcal{H})$, then $e^{\lambda_{j}(\dot{u})}=1$ for every $j$, that is, $(o, \dot{u}) \in \mathfrak{h}$ is exptrivial.

Proof. Lemma 7.1 and Corollary 7.2 part 1. apply. Let $w=\sum_{j} w_{j}$ with $w_{j} \in V_{j}$. By eq (1) with $\left(v_{1}, u_{1}\right)=(v, u)$ and $\left(v_{2}, u_{2}\right)=(w, 0),(v, u)(w, 0)=\left(v+\sum e^{\lambda_{j}(\dot{u})} w_{j}, u\right)$. Similarly, $(w, 0)(v, u)=(w+v, u)$. These computations can only agree for all choices of $w$ if $e^{\lambda_{j}(\dot{u})}=1$ for all $j$.

Corollary 7.4. Suppose that in Lemma 7.1, $V=X \oplus Y$ where $X$ and $Y$ are invariant subspaces for all $\phi(\dot{u})$. Let $\dot{u}_{1} \in U$ satisfy $\phi\left(\dot{u}_{1}\right)<x>=2 \pi n x(0 \neq n \in \mathbb{Z})$ for all $x \in X$. Suppose that $\dot{u}_{2} \in U$ is such that Range $\left(\phi\left(\dot{u}_{2}\right)\right) \subsetneq X$. If $\dot{u}=\dot{u}_{1}+\dot{u}_{2}$, $\dot{y} \in X$, and $\dot{y} \notin \operatorname{Range}\left(\phi\left(\dot{u}_{2}\right)\right)$, then the first component of $\exp _{\mathcal{H}}(\dot{v}, \dot{u})$ ) (that is, the first component on the right in eq. (2) with $t=1)$ cannot equal the first component of $\exp _{\mathcal{H}}(\dot{y}, 0)$ for any choice of $\dot{v}$.

Proof. Corresponding to $V=X \oplus Y$ one has $\mathcal{V}=\mathcal{X} \oplus \mathcal{Y}$. Since $\left[\dot{u}_{1}, \dot{u}_{2}\right]=0$, if $v=v_{x}+v_{y}$ with $v_{x} \in \mathcal{X}$ and $v_{y} \in \mathcal{Y}$, then the projection of the first component of $\exp _{\mathcal{H}}((\dot{v}, \dot{u}))$ onto $\mathcal{X}$ is

$$
\begin{gathered}
\int_{0}^{1} \operatorname{Exp}_{V}\left(s \lambda\left(\dot{u}_{1}\right) I_{X}\right) \operatorname{Exp}_{V}\left(s \phi\left(\dot{u}_{2}\right)\right)<v_{x}>d s= \\
\left.\int_{0}^{1} e^{2 \pi i n s} v_{x} d s+\int_{0}^{1} e^{2 \pi i n s}\left(\operatorname{Exp}_{V}\left(s \phi\left(\dot{u}_{2}\right)\right)\right)-I\right)<v_{x}>d s .
\end{gathered}
$$

The first term on the lower line vanishes since $n \neq 0$ and the second is in the subspace Range $\left(\phi\left(\dot{u}_{2}\right)\right)$, so the sum cannot be $y$ for any choice of $v$. But the first component of $h=\exp _{\mathcal{H}}(\dot{y}, 0)$ is $y$. 


\section{Proof of Theorem 1}

Suppose that $F$ is a complex exponential Lie group (excluding the trivial case where $F$ is nilpotent). It is to be proved that the center $Z(F)$ is connected, but first the results of the preceding sections will be used to show that no generality is lost by assuming that $F$ satisfies some restrictive conditions. That is, $F$ can be replaced by other groups having more special properties, but which preserve exponentiality and the connectivity of $Z(F)$. By first applying Corollary 4.1, then renaming $F_{*}$ as $F$, it can be assumed that:

(1) $F$ is exponential.

(2) The center of the Lie algebra is trivial (hence the adjoint representation of the Lie algebra is faithful).

(3) $Z(F)$ is discrete.

(4) The Lie algebra of $F$ is semi-algebraic (by Corollary 5.1).

Let $G$ be the simply connected covering group of $F$ so that $F=G / Y \pi_{Y}: G \longrightarrow F$, where $Y \subseteq Z(G)$. It is to be shown that $Z(F)$ is trivial (or $Y=Z(G)$ ). The Levi decomposition gives $G=R \ltimes S$ where $S$ is semisimple and $R$ is solvable and the radical of $\mathfrak{g}, \mathfrak{r}=\mathfrak{n} \oplus \mathfrak{a}$, is a semidirect sum where $\mathfrak{a}$ is abelian and acts semisimply on $\mathfrak{n}$, which is nilpotent.

Next it will be shown that no generality is lost by assuming that $\mathfrak{n}$ is abelian. Let $[N, N] \subseteq R \subseteq G$ be the connected normal subgroup with $[\mathfrak{n}, \mathfrak{n}]$ as its Lie algebra, and $\psi: G \longrightarrow G /[N, N]$ the natural map. By $[6$, pp. 135-136], $[N, N]$ is closed and $G /[N, N]$ is simply connected. Moreover, the restriction of $\psi$ to $Z(G)$ is injective. This is the case because by Proposition 3.3 the elements of $[N, N] \subseteq \exp _{N}(\mathfrak{n})$ cannot belong to $Z(G)$. Noting that $\psi(Y) \subseteq Z(G /[N, N])$, defining $F^{b}=(G /[N, N]) / \psi(F)=$ $(G / Y) / \pi_{Y}([N, N])$, and $\psi_{N}(f)$ as the coset $f \pi_{Y}([N, N])$ one has the diagram below. It is commutative because the kernel of either homomorphism from $F$ to $F^{b}$ is $Y \cdot[N, N]$.

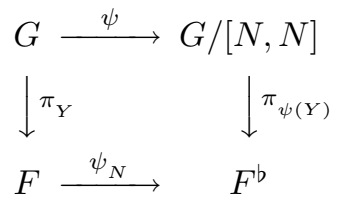

Now we observe that $G$ and $F$ can for purposes of our proof be replaced respectively by $G /[N, N]$ and $F^{b}=(G /[N, N]) / \psi(Y)$. That is, these groups retain the properties $(1),(2)$, and (3) listed above. Condition (1) follows from Proposition 4.1 (1); (2) follows from Lemma $2.3 ;(3)$ is clear and, in fact, the injectivity of $\psi$ on $Z(G)$ gives $Z(G) \cong Z(\psi(G /[N, N]))$ and also $Y \cong \psi(Y)$. Condition (4) can also be verified easily, since the induced map $d \psi$ on the lie algebra just annihilates the ideal $[\mathfrak{n}, \mathfrak{n}]$. The point of this replacement is that with it one obtains the additional condition that $\mathfrak{n}$ is abelian rather than merely nilpotent. For the remainder of the proof it can be assumed that $F$ and $G$ have the properties (1) through (4) above and in addition that $\mathfrak{n}$ is abelian.

If $S$ is trivial, $G$ is solvable and the desired conclusion was derived in [21]. If $S$ is not trivial. By Weyl's theorem the representation of the semisimple Lie algebra $\mathfrak{s}$ on $\mathfrak{r}$ is completely reducible. This means $\mathfrak{r}=W_{0} \oplus W_{1} \oplus \cdots \oplus W_{q}$, where $\mathfrak{s}$ acts trivially on $W_{0}$ and for each $j>0$ the representation of at least one simple factor of $\mathfrak{s}$ 
is faithful on the minimal invariant subspace $W_{j}$. Since $F$ is semi-algebraic, $[\mathfrak{s}, \mathfrak{a}]=0$, so $\mathfrak{a} \subseteq W_{0}$ and the actions of $\mathfrak{s}$ and $\mathfrak{a}$ on $\mathfrak{n}$ commute. Then by Schur's Lemma, every $\dot{a} \in \mathfrak{a}$ acts by a scalar multiple of the identity on each $W_{j}$ for $j>0$.

It is obvious from Proposition 3.3 that if $\mathfrak{t}$ is a Cartan subalgebra of $\mathfrak{g}$ the images of elements of $\mathfrak{t}$ under $\exp _{G}$ that are exptrivial are contained in $Z(G)$. The following assertions, which are easy to verify using the remarks in the parentheses, show that the converse is true and give some details about how the the exponential map acts on parts of $\mathfrak{t}$. For the sake of brevity, elements $x$ in a Lie algebra will be called exptrivial if $a d_{x}$ is exptrivial.

(1) If $\mathfrak{h}$ is a Cartan subalgebra of $\mathfrak{s}$, then the direct sum $\mathfrak{t}=\mathfrak{a} \oplus \mathfrak{h}$ is a Cartan subalgebra of $\mathfrak{g}$. (It was assumed at the beginning of the proof that $F$ was not nilpotent. $[\mathfrak{s}, \mathfrak{a}]=0$ implies that $\mathfrak{t}$ is abelian. If $\dot{m}=(\dot{n}, \dot{a}, \dot{s})$ is an element of the normalizer of $\mathfrak{t},[\dot{m}, \dot{h}] \in \mathfrak{t}$ for all $\dot{h} \in \mathfrak{h}$ implies that $\dot{n} \in \mathfrak{n} \cap W_{0}$. But here $\mathfrak{z}(\mathfrak{g})=0$, so if $[\dot{m}, \dot{b}] \in \mathfrak{t}$ for all $\dot{b} \in \mathfrak{a}$ with $\dot{n} \in \mathfrak{n} \cap W_{0}, \dot{n}=0$. Therefore, $\dot{m} \in \mathfrak{t}$, which is its own normalizer.)

(2) If $\dot{f} \in \mathfrak{g}$ is exptrivial, then there is an elementary automorphism $\alpha$ of $\mathfrak{g}$ (that is, an automorphism that is a finite composition of automorphisms $e^{a d(\eta)}$ with $\eta \in \mathfrak{n}$ ) such that $\alpha(\dot{f})=\dot{g} \in \mathfrak{t}$. (Corollary 7.1, [1, VII, $\S 3.2$, Theorem $1]$ ). Also since $Z(G)$ is discrete and the set of elementary automorphisms is connected $\exp _{G}(\dot{f})=\exp _{G}(\dot{g})$.

(3) Every $\mathfrak{g}$-exptrivial (i.e. as an element of $\mathfrak{g}$ ) element of $\mathfrak{r}$ has a conjugate with respect to $R=\operatorname{Rad}(G)$ in $\mathfrak{A}=\{\dot{a} \in \mathfrak{a}: 2 \pi i \dot{a}$ is $\mathfrak{g}$-exptrivial $\}$. (By [1, VII, $\S 3.4$, Theorem 3] and Proposition 7.1 a r-exptrivial element has a conjugate in a Cartan subalgebra of $\mathfrak{r}$ containing $\mathfrak{a}$. But by the argument in (1) if $\dot{n}+\dot{a} \in \mathfrak{n} \oplus \mathfrak{a}$ is $\mathfrak{g}$-exptrivial, $\mathfrak{z}(\mathfrak{g})=0$ implies that $\dot{n}$ is trivial.)

(4) If $\mathfrak{h}$ is a Cartan subalgebra of $\mathfrak{s}, \dot{s} \in \mathfrak{h}$ is $\mathfrak{s}$-exptrivial if it is $\mathfrak{g}$-exptrivial. So if $\mathfrak{S}=\{\dot{s} \in \mathfrak{h}: 2 \pi i \dot{s}$ is $\mathfrak{g}$-exptrivial $\}$, every $\mathfrak{g}$-exptrivial element of $\mathfrak{s}$ has a conjugate (with respect to $S$ ) in $\mathfrak{S}$.

(5) $(\dot{a}, \dot{s}) \in \mathfrak{t}$ is exptrivial in $\mathfrak{g}$ if $(\dot{a}, 0)$ and $(0, \dot{s})$ are exptrivial in $\mathfrak{g} .([\mathfrak{s}, \mathfrak{a}]=0)$

(6) $Z(G) \subseteq E(G)$. (If $r \in Z(R) \cap Z(G)$, Corollary 7.3 shows that $r$ has a $R$ conjugate in $E(R)$, and by Proposition 3.3 this conjugate must be the image of an exptrivial element of $\dot{r} \in \mathfrak{r}$. By the argument in (3), $\dot{r}$ can be chosen in $\mathfrak{a} \oplus \mathfrak{z}(\mathfrak{r})$. But $\exp _{R}(\mathfrak{a})=\exp _{R}(\mathfrak{a} \oplus \mathfrak{z}(\mathfrak{r}))$, so $\dot{r}$ can be chosen in $\mathfrak{a}$. $Z(S) \subseteq E(S)$ by the remark after the proof of Proposition 3.3. Then if $g=(r, s) \in Z(G)=$ $Z(R \ltimes S), g$ commutes with all elements $\left(r^{\prime}, 0\right),\left(0, s^{\prime}\right) \in G$, so $s \in Z(S) \subseteq$ $E(S)$ and $r \in E(A)$. Then $g=\exp _{G}(\dot{r}) \exp _{G}(\dot{s})=\exp _{G}(\dot{r}+\dot{s}) \in E(G)$ since $[\mathfrak{a}, \mathfrak{s}]=0$.

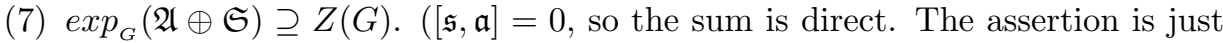
an interpretation of the argument in (6) since Proposition 3.3 and (5) give $\left.\exp _{G}(\mathfrak{A} \oplus \mathfrak{S}) \subseteq Z(G), \exp _{G}(\mathfrak{A} \oplus \mathfrak{S})=Z(G).\right)$

(8) Let $\mathfrak{A}_{F}=\left\{\dot{a} \in \mathfrak{A}: \exp _{F}(\dot{a}, 0)=1_{F}\right\}, \mathfrak{S}_{F}=\left\{\dot{s} \in \mathfrak{S}: \exp _{F}(0, \dot{s})=1_{F}\right\}$, and $\exp _{G}\left(\mathfrak{A}_{F} \oplus \mathfrak{S}_{F}\right)=Y$. If $\mathfrak{A}_{F}=\mathfrak{A}$ and $\mathfrak{S}_{F}=\mathfrak{S}$, then $Z(G)=Y$ and $Z(F)$ is connected. (7)

It is easy to see using Lemma 6.1 (or the Theorem of Lai-Wüstner) that if $F$ is exponential $\mathfrak{S}_{F}=\mathfrak{S}$. Therefore by (8) in the list above, if it can be shown that $\mathfrak{A}_{F}=\mathfrak{A}$, it will follow that $Z(F)$ is connected. 
Suppose that $\mathfrak{A}_{F} \subsetneq \mathfrak{A}$ and let $\dot{a} \in \mathfrak{A} \backslash \mathfrak{A}_{F}$ be a representative of a cyclic coset of the quotient $\dot{a} \mathfrak{A}_{F} \in \mathfrak{A} / \mathfrak{A}_{F}$. Applying Lemma 2.1 to the adjoint action of $\mathfrak{a}$ on $\mathfrak{n}$ one finds a $\lambda_{k}$ such that $\lambda_{k}(\dot{a}) \neq 0$ for every choice of $\dot{a}$ in the coset.

There are then two possibilities:

(1) $\mathfrak{n}\left(\lambda_{k}\right) \supseteq W_{j}$ for some $j>0$, or

(2) $\mathfrak{n}\left(\lambda_{k}\right) \subseteq W_{0} \cap \mathfrak{n}$.

These are not mutually exclusive, but they are exhaustive, since if neither (1) nor (2) were to hold $[\mathfrak{s}, \dot{a}]=0$ would imply $a d_{\dot{a}} \equiv 0$ and $\dot{a} \in \mathfrak{z}(\mathfrak{g})$. But here $\mathfrak{z}(\mathfrak{g})=0$.

If (1) holds, Corollary 7.4 can be applied taking $\mathcal{H}=\mathcal{V} \ltimes_{\Phi} \mathcal{U} \subseteq G$ as the simply connected solvable Lie group with Lie algebra $V \oplus U$, where $V=\mathfrak{n}$ and $U=\mathfrak{a} \oplus \mathbb{C} \dot{w}$ with $\dot{w} \in \mathfrak{h} \subseteq \mathfrak{s}$ as in Lemma 6.1 . It can be assumed that $\left[\dot{w}, \dot{v}_{j}\right] \neq 0$ for some $\dot{v}_{j} \in W_{j}$, since some nontrivial simple factor of $\mathfrak{s}$ must act nontrivially on $W_{j}$. Let $V=X \oplus Y, X=W_{j}$, and $Y=W_{J}^{p}$, a complementary $\mathfrak{t}=\mathfrak{a} \oplus \mathfrak{s}$-invariant subspace of $\mathfrak{n}$, and set $\dot{u}_{1}=\dot{a}$. Then the corollary shows that there is an element $y \in \mathcal{H} \backslash E(\mathcal{H})$, because by [1, I $\S 6.3$, Theorem 3] $a d_{\dot{w}}$ must be nilpotent (hence not surjective) on $W_{j}$. The intersection of the Lie algebra of $\mathcal{H}$ with the Levi factor $\mathfrak{s}$ is just $\mathbb{C} \dot{w}$ and by Lemma 6.1 , any $\dot{g}=(\dot{r}, \dot{s}) \in \mathfrak{g}$ such that $\exp _{G}(\dot{g})=(y, a, w) \in G$ must satisfy $\dot{s}=\dot{w}$. But such an element would be in $E(\mathcal{H})$, contradicting what has been proved. Hence $g=(y, a, w) \notin E(G)$ for any choice of $\dot{a}$. If $F$ were exponential one of the preimages of $\pi_{Y}(g)$ would have to be in $E(G)$, so $F$ is not exponential.

If (2) holds, apply Corollary 7.2, taking $U=\mathfrak{a}, V=W_{0} \cap \mathfrak{n}$, and $\mathcal{H}=\mathcal{V} \ltimes \mathcal{U}$. Then the conclusion shows that unless $\dot{a}$ is trivial there is an element $g \in \mathcal{H}$ that is not in $E(\mathcal{H})$. But $g$ cannot even be in $E(G)$ because $\exp _{G}(\mathfrak{s})$ and $\exp _{G}\left(\mathfrak{n} \ominus\left(W_{0} \cap \mathfrak{n}\right)\right)$ do not produce anything nontrivial in $\mathcal{H}$. (Here $\mathfrak{n} \ominus\left(W_{0} \cap \mathfrak{n}\right)$ ) denotes a subspace of $\mathfrak{n}$ that is complementary to $W_{0} \cap \mathfrak{n}$ and is invariant under the adjoint action of $\mathfrak{t}=\mathfrak{a} \oplus \mathfrak{s}$.) This completes the proof of Theorem 1.

\section{References}

[1] N. Bourbaki, Lie Groups and Lie Algebras, Springer Verlag.

[2] A. Borel, Linear Algebraic Groups, Second Enlarged Addition, Springer-Verlag, New York, Berlin, Heidelberg, 1991.

[3] P. Chatterjee, On the Surjectivity of Power Maps of Algebraic Groups in Characteristic Zero, Math. Research Letters, 9 (2002), 741-756.

[4] J. Dixmier, L'application exponentielle dans les groupes de Lie résolubles, Bull. Soc. Math. France, 85 (1957), 113-121.

[5] D. Djokovic and K.H. Hofmann, The surjectivity question for the exponential function in real Lie groups: A status report, Journal of Lie Theory, 7 (1997), 171-199.

[6] G. Hochschild, The Structure of Lie Groups Holden-Day, San Francisco, 1965.

[7] J. Humphries, Linear Algebraic Groups Springer-Verlag, New York, Berlin, Heidelberg, 1981.

[8] N. Jacobson, Lie Algebras, Dover Publishing Co.

[9] H. L. Lai, Surjectivity of exponential map on semisimple Lie groups, J. Math. Soc. Japan, 29 (1977), 303-325.

[10] H. L. Lai, Exponential map of a center-free complex simple Lie group, Osaka J. Math. 27 (1978), 553-560.

[11] D.H. Lee, The Structure of Complex Lie Groups Chapman \& Hall/CRC, Research Notes in Mathematics No. 429, Boca Raton, London, New York, Wash. D.C., 2002.

[12] M. Moskowitz, Exponentiality of algebraic groups, Journal of Algebra, 186 (1996), 20-31.

[13] M. Moskowitz and R. Sacksteder, The exponential map and differential equations on real Lie groups, J. of Lie Theory, Vol 13 (2003), 291-306. 
[14] M. Moskowitz and M. Wüstner, Exponentiality of certain real solvable Lie groups, Canad. Math. Bull., 41 (1998), 368-373.

[15] M. Moskowitz and M. Wüstner, Contributions to real exponential Lie groups, Monatshefte für Mathamatik, 130 (2000), 29-47.

[16] G.D. Mostow, Fully reducible subgroups of algebraic groups, Amer. J. Math., 78 (1956), 200-221.

[17] R. Steinberg, Conjugacy Classes in Algebraic Groups, Springer Lecture Notes in Mathematics \#366.

[18] E.B. Vinberg, A Course in Algebra, A.M.S. Graduate Studies in Mathamatics \#56.

[19] Frank W. Warner, Foundations of Differentiable Manifolds and Lie Groups, Scott, Foresman and Company, Glenville, IL 1971.

[20] M. Wüstner, On the surjectivity of the exponential function of complex algebraic, complex semisimple and complex splittable Lie groups, Journal of Algebra, 184 (1996), 1082-1092.

[21] M. Wüstner, On the surjectivity of the exponential function of solvable Lie groups Math. Nachr., 192 (1998), 255-266

Ph.D Program in Mathematics, CUNy Graduate Center, 365 Fifth Ave., New York, Ny 10016

E-mail address: martin.moskowitz@gmail.com 